\title{
STRUCTURE AND MAGNETIC PROPERTIES OF A NICKEL-BASED SUPERALLOY AFTER DEFORMATION
}

\author{
D. I. Davydov*, S. V. Afanasiev, V. P. Pilyugin, D. A. Shishkin, P. B. Terent'ev \\ M.N. Miheev Institute of Metal Physics of Ural Branch of Russian Academy of Sciences, \\ 18 S. Kovalevskoy st., Ekaterinburg, Russian Federation

\begin{abstract}
*Corresponding author. E-mail: davidov@imp.uran.ru; address for correspondence: 18, ul. S. Kovalevskoy, 620990,
\end{abstract} \\ Ekaterinburg, Russian Federation. Tel.: +7 343 3783711; fax: +7 3433745244
}

The structure and magnetic properties of ChS-70 nickel-based superalloy samples have been compared after different types of deformation. The different behavior of the magnetic properties is governed by the difference in the structure resulting from deformation. High-temperature tensile tests and shear deformation under high pressure have been performed for ChS-70 alloy samples. Samples cut from different parts of a turbine blade after operation under forced conditions have been examined. The deformation of the Chs-70 alloy under operation increases magnetic susceptibility, which is due to the formation of complex defects of the crystalline structure inside the intermetallic particles $\left(\mathrm{Ni}_{3} \mathrm{Al}\right)$. Dynamical recovery during the high-temperature tensile tests leads to relaxation, elimination of crystal lattice defects, and the magnetic properties remain unchanged. Shear under high pressure causes a nanocrystalline structure in the alloy, a significant decrease in the degree of the long-range order in the intermetallic phase and the preservation of the paramagnetic state.

Keywords: structure, deformation, magnetic properties, stacking faults, nickel-based alloy.

DOI: $10.17804 / 2410-9908.2016 .1 .016-028$

\section{References}

1. Stoloff N.S. Physical and mechanical metallurgy of $\mathrm{Ni}_{3} \mathrm{Al}$ and its alloys. International Materials Reviews, 1989, vol. 34, iss. 4, pp. 153-183.

2. Deryagin A.I., Zavalishin V.A., Saragadze V.V., Efros B.M. Formation of nanosized ferromagnetic phases during plastic deformation and subsequent annealing of stable austenitic steels. Russian Journal of Nondestructive Testing, 2007, vol. 43, iss. 7, pp. 427-435. DOI: $10.1134 / \mathrm{S} 1061830907070029$.

3. Rhee Joo Yull, Kudryavtsev Y.V., Lee Y.P. Optical, magneto-optical, and magnetic properties of stoichiometric and off-stoichiometric $\gamma^{\prime}$-phase $\mathrm{Ni}_{3} \mathrm{Al}$ alloys. Physical Review B, 2003, vol. 68, iss. 4, pp. 045104-1-045104-8. DOI: 10.1103/PhysRevB.68.045104.

4. Idzikowski Bogdan, Kudryavtsev Yuri V., Hyun Young-Hoon, Lee Young-Pak, Klenke Jens. Magnetic effects of structural disorder in the itinerant ferromagnet $\mathrm{Ni}_{3} \mathrm{Al}$ studied by magnetic and neutron methods on stoichiometric and off-stoichiometric samples. Journal of Alloys and Compounds, 2006, vol. 423, iss. 1-2, pp. 267-273. DOI: 10.1016/j.jallcom.2006.01.088.

5. Zeng Q., Baker I. The effect of local versus bulk disorder on the magnetic behavior of stoichiometric $\mathrm{Ni}_{3} \mathrm{Al}$. Intermetallics, 2007, vol. 15, iss. 3, pp. 419-427. DOI: 10.1016/j.intermet.2006.08.010.

6. Umakoshi Y., Hiroyuki Y., Toshifumi Y. Quantitative analysis of $\gamma$ (gamma) precipitate in cyclically deformed $\mathrm{Ni}_{3}(\mathrm{Al}, \mathrm{Ti})$ single crystals using magnetic technique. Proc. MRS Fall Meeting, November 28-December 3, 2004, Boston, USA, 2004, vol. 842, pp. 2.3.1-2.3.6.

7. Stepanova N.N., Davydov D.I., Nichipuruk A.P., Rigmant M.B., Kazantseva N.V., Vinogradova N.I., Pirogov A.N., Romanov E.P. The structure and magnetic properties of a heatresistant nickel-base alloy after a high-temperature deformation. The Physics of Metals and Metallography, 2011, vol. 112, no. 3, pp. 309-317. DOI: 10.1134/S0031918X11030288. 
8. Ray A.K., Singh S.R., Swaminathan J., Roy P.K., Tiwari Y.N., Bose S.C., Ghosh R.N. Structure property correlation study of a service exposed first stage turbine blade in a power plant. Materials Science and Engineering: A, 2006, vol. 419, iss. 1-2, pp. 225-232. DOI: $10.1016 /$ j.msea.2005.12.030.

9. Levit V.I., Smirnov M.A. Vysokotemperaturnaya termomekhanicheskaya obrabotka austenitnykh stalei $i$ splavov [High-Temperature Thermomechanical Treatment of Austenitic Steels and Alloys]. Chelyabinsk, CHGTU Publ., 1995, 276 p. (In Russian).

10. Skudnov V.A., Tarasenko Yu.P., Berdnik O.B. Selection of optimal operating temperature for ChS70-VI and ChS88U-VI nickel-based alloys in terms of synergetics. Tekhnologiya metallov, 2008, no. 12, pp. 16-20. (In Russian).

11. Korznikov A.V., Tram G., Dimitrov O., Korznikova G.F., Idrisova S.R., Pakiela Z. The mechanism of nanocrystalline structure formation in $\mathrm{Ni}_{3} \mathrm{Al}$ during severe plastic deformation. Acta Materialia, 2001, vol. 49, iss. 4, pp. 663-671. DOI: 10.1016/S1359-6454(00)00345-1.

12. Tyumentsev A.N., Tretyak M.V., Pinzhin Yu.P., Korotaev A.D., Valiev R.Z. Evolution of defect substructure in the $\mathrm{Ni}_{3} \mathrm{Al}$ alloy in the course of severe plastic deformation by torsion under pressure. Fizika Metallov i Metallovedenie, 2000, vol. 90, iss. 5, pp. 44-54. (In Russian).

13. Kazantseva N.V., Pilyugin V.P., Zavalishin V.A., Stepanova N.N. Effect of a nanosized state on the magnetic properties of $\mathrm{Ni}_{3}(\mathrm{Al}, \mathrm{Fe})$ and $\mathrm{Ni}_{3}(\mathrm{Al}, \mathrm{Co})$. The Physics of Metals and Metallography, 2014, vol. 115, iss. 3, pp. 243-247. DOI: 10.1134/S0031918X14030053. 
Подана в журнал: 23.12 .2015

УДК 620.18:537.622:620.17:669.017

DOI: $10.17804 / 2410-9908.2016 .1 .016-028$

\title{
СТРУКТУРА И МАГНИТНЫЕ СВОЙСТВА ЖАРОПРОЧНОГО НИКЕЛЕВОГО СПЛАВА ПОСЛЕ ДЕФОРМАЦИИ
}

\author{
Д. И. Давыдов*, С. В. Афанасьев, В. П. Пилюгин, Д. А. Шишкин, П. Б. Терентьев \\ Федеральное государственное бюджетное учреждение науки Институт физики металлов имени \\ М.Н. Михеева Уральского отделения Российской академии наук, ул. С.Ковалевской, 18, 620990, \\ Екатеринбург, Российская Федерации
}

\begin{abstract}
*Ответственный автор. Электронная почта: davidov@imp.uran.ru; адрес для переписки: ул. С. Ковалевской, 18, 620990, Екатеринбург, Российская Федерация. Телефон: +7 343 378-37-11; факс: +7 343 374-52-44
\end{abstract}

Проведено сравнение структуры и магнитных свойств образцов жаропрочных никелевых сплавов ЧС-70 после различных видов деформации. Различный характер изменения магнитных свойств определяется различием структуры, сформированной в результате деформации.

Для ЧС-70 проведены высокотемпературные испытания на растяжение; деформация сдвигом под высоким давлением, исследованы образцы, вырезанные из разных частей турбинной лопатки после эксплуатации по форсированному режиму. Деформация сплава ЧС-70 в ходе его эксплуатации приводит к возрастанию магнитной восприимчивости, связаному с формированием комплексов дефектов кристаллического строения внутри частиц интерметаллидной фазы $\left(\mathrm{Ni}_{3} \mathrm{Al}\right)$. Динамический возврат при высокотемпературных испытаниях на растяжение приводит к релаксации, устранению дефектов кристаллической решетки, и магнитные свойства сплава не изменяются. Сдвиг под высоким давлением приводит к образованию в сплаве нанокристаллической структуры, к значительному уменьшению степени дальнего порядка интерметаллической фазы и сохранению парамагнитного состояния.

Ключевые слова: структура, деформация, магнитные свойства, дефекты упаковки, никелевые сплавы.

\section{1. Введение}

Для изготовления турбинных лопаток, наиболее ответственных деталей газотурбинных двигателей применяются жаропрочные никелевые сплавы. Основной упрочняющей фазой этих сплавов является интерметаллическое соединение $\mathrm{Ni}_{3} \mathrm{Al}$, объемная доля которого может достигать 90 \%. Интерметаллическое соединение $\mathrm{Ni}_{3} \mathrm{Al}$ имеет ГЦК кристаллическую решетку со сверхструктурой $L 1_{2}$ и существует в узком интервале концентраций вблизи 75 ат. \% Ni. Интерметаллид характеризуется высокой степенью дальнего порядка, которая сохраняется вплоть до температуры плавления [1]. При больших пластических деформациях наблюдается явление деформационно-индуцированного магнетизма, присущее широкому кругу интерметаллидных соединений, в том числе и $\mathrm{Ni}_{3} \mathrm{Al}$. Оно проявляется как образование ферромагнитных кластеров в исходно парамагнитной матрице. Близким по сути своей явлением может быть образование в исходно парамагнитных твердых растворах ферромагнитных кластеров на основе локальных неоднородностей состава. Такие неоднородности создаются потоками точечных дефектов в процессе деформации [2]. Это как раз тот случай, когда зарождение и рост ферромагнитных кластеров с помощью магнитных методов регистрируются на стадии предвыделений. В то время как при дифракционных исследованиях (рентгеновских и электронно-микроскопических) изучение предвыделений затруднено, поскольку такие кластеры не выявляются как самостоятельная фаза. 
Магнитные свойства интерметаллического соединения $\mathrm{Ni}_{3} \mathrm{Al}$ зависят от ближайшего окружения атомов никеля и чувствительны к составу сплава в пределах области гомогенности. $\mathrm{Ni}_{74} \mathrm{Al}_{26}$ остается в парамагнитном состоянии до $4 \mathrm{~K}$, тогда как $\mathrm{Ni}_{75} \mathrm{Al}_{25}$ является слабым ферромагнетиком с температурой Кюри 41К [3].

При отходе от стехиометрического состава в $\mathrm{Ni}_{3} \mathrm{Al}$ были обнаружены несколько температур Кюри (магнитное расслоение), одна из которых смещена в сторону комнатной температуры [4]. Появление магнитного расслоения авторы объясняют флуктуацией химического состава при образовании двухфазной смеси из кластеров слабо упорядоченной $\gamma$-фазы и упорядоченной $\gamma^{\prime}$-фазы. Магнитные свойства $\mathrm{Ni}_{3} \mathrm{Al}$ также чувствительны к деформации. В интерметаллидах, в том числе $\mathrm{Ni}_{3} \mathrm{Al}$, после холодной деформации прокаткой также наблюдалось образование магнитных кластеров [5]. При циклической деформации исходного парамагнитного никелевого жаропрочного сплава было обнаружено суперпарамагнитное состояние [6].

Практический интерес представляет исследование жаропрочных сложнолегированных никелевых сплавов, применяемых для производства турбинных лопаток. Никелевые жаропрочные сплавы структурно состоят из твердого раствора на основе никеля ( $\gamma$-фаза, ГЦК); упрочняющей $\gamma$-фазы $\left(\mathrm{Ni}_{3} \mathrm{Al}\right)$ и небольшого количества карбидов. Сплавы при температурах выше комнатной находятся в парамагнитном состоянии и имеют низкое значение магнитной восприимчивости $\chi$ порядка $10^{-4}$.

Каждый сплав имеет предельное значение рабочей температуры. Работа деталей по стандартному режиму (на 50-100 ${ }^{\circ} \mathrm{C}$ ниже предельной температуры) в течение всего срока эксплуатации не сопровождается изменением магнитного состояния. В [7] впервые наблюдалось увеличение магнитной восприимчивости никелевого жаропрочного сплава после его высокотемпературной деформации при повышенной (практически до предельной) температуре. Магнитная восприимчивость менялась от $2 \cdot 10^{-4}$ в исходном состоянии до значения $360 \cdot 10^{-4}$ так, как если бы появился ферромагнитный вклад в намагниченность исходно парамагнитного образца. При этом общепринятые представления о деформации жаропрочных никелевых сплавов не предполагают фазовых переходов, связанных с образованием какихлибо новых фаз, карбидные превращения приводят к образованию карбида $\mathrm{Me}_{23} \mathrm{C}_{6}$ вместо карбида $\mathrm{MC}$, но оба они являются парамагнитными и не влияют на появление ферромагнитного вклада.

Для понимания структурных механизмов влияния деформации на магнитные свойства представляет интерес сопоставление структуры жаропрочных никелевых сплавов, полученной в результате различных деформационных воздействий.

В работе проведен сравнительный анализ структуры и магнитных свойств сплава ЧС70 после трех видов деформации: высокотемпературных испытаний на активное растяжение, после деформации сдвигом под высоким давлением и высокотемпературной деформации в качестве материала турбинной лопатки после эксплуатации по форсированному режиму.

\section{2. Материал и методика}

Химический состав сплава ЧС-70 (ХН58КВТЮМБЛ) представлен в табл. 1.

Таблица 1 - Химический состав сплава ЧС-70

\begin{tabular}{|c|c|c|c|c|c|c|c|c|}
\hline $\mathrm{C}$ & $\mathrm{Cr}$ & $\mathrm{Mo}$ & $\mathrm{Nb}$ & $\mathrm{Ti}$ & $\mathrm{Al}$ & $\mathrm{W}$ & $\mathrm{Co}$ & $\mathrm{Fe}$ \\
\hline 0,10 & 15,4 & 1,5 & 0,25 & 5,0 & 3,5 & 3,6 & 10,6 & 0,8 \\
\hline
\end{tabular}

Для оценки исходного состояния сплава ЧС-70 использован образец после стандартной термообработки - ступенчатый отжиг: $1170{ }^{\circ} \mathrm{C}, 4$ ч; охлаждение с печью; $1050{ }^{\circ} \mathrm{C}, 4$ ч; охлаждение на воздухе; $850{ }^{\circ} \mathrm{C}, 16$ ч (ТУ 14-1-3658-83). Объемная доля упрочняющей интерметаллидной фазы $\left(\mathrm{Ni}_{3} \mathrm{Al}\right)$ составляет 40 об. \%.

Davydov D.I. et al. / Structure and magnetic properties of a nickel-based superalloy after deformation. 
Турбинная лопатка из сплава ЧС-70 эксплуатировалась в промышленных условиях по экспериментальному форсированному режиму при повышенной до $880{ }^{\circ} \mathrm{C}$ температуре относительно стандартного режима $\left(800^{\circ} \mathrm{C}\right)$ и увеличенной скорости вращения турбины. Предельной температурой эксплуатации сплава является $900{ }^{\circ} \mathrm{C}$. Лопатка стояла на промышленной установке в течение 9390 ч (13 месяцев), 17 пусков. Образцы вырезали из различных частей лопатки на электроискровом станке и повергали электрополировке.

Испытания на растяжение образцов сплава ЧС-70 до разрушения проводили на машине для определения механических свойств материалов Инстрон в температурном интервале $880-920^{\circ} \mathrm{C}$, скорость нагружения составляла 0,05 мм/мин.

Образцы сплава ЧС-70 диаметром 5 мм и толщиной 0,3 мм деформировали методом сдвига под высоким давлением 8 ГПа при температуре $20^{\circ} \mathrm{C}$ с углом поворота наковальни до десяти оборотов. Скорость вращения наковальни составляла $(\omega)$ 0,3 оборота в минуту. Твердость деформированных образцов измеряли на приборе ПМТ-3 при нагрузке 0,5 Н по двум взаимно перпендикулярным диаметрам.

Исследования структуры проведены в ЦКП «Испытательный центр нанотехнологий и перспективных материалов» ИФМ УрО РАН с использованием просвечивающего электронного микроскопа JEM-200CX. Для магнитных измерений был использован вибрационный магнитометр Lake Shore 7407. Измерения проведены в магнитных полях до 17 кЭ на частоте 82 Гц, амплитуда вибраций составляла 1,5 мм. Относительная погрешность измерений не более $1 \%$. Для измерения АС-восприимчивости использовалась установка, основанная на методе скомпенсированного трансформатора в интервале температур от 90 до 360 K.

\section{3. Результаты и обсуждение}

Литая поликристаллическая лопатка из сплава ЧС-70 состоит из массивного замка и тонкого пера. Распределение напряжений в работающей лопатке неоднородно и подробно описано в работе [8]. Неоднородность в распределении напряжений приводит к тому, что в различных участках лопатки структурное состояние после эксплуатации различно (рис. 1).

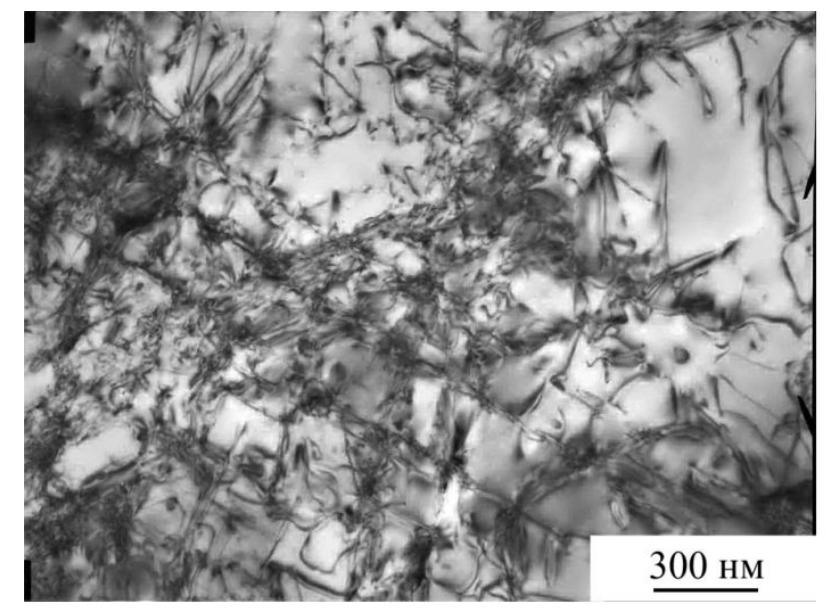

$a$

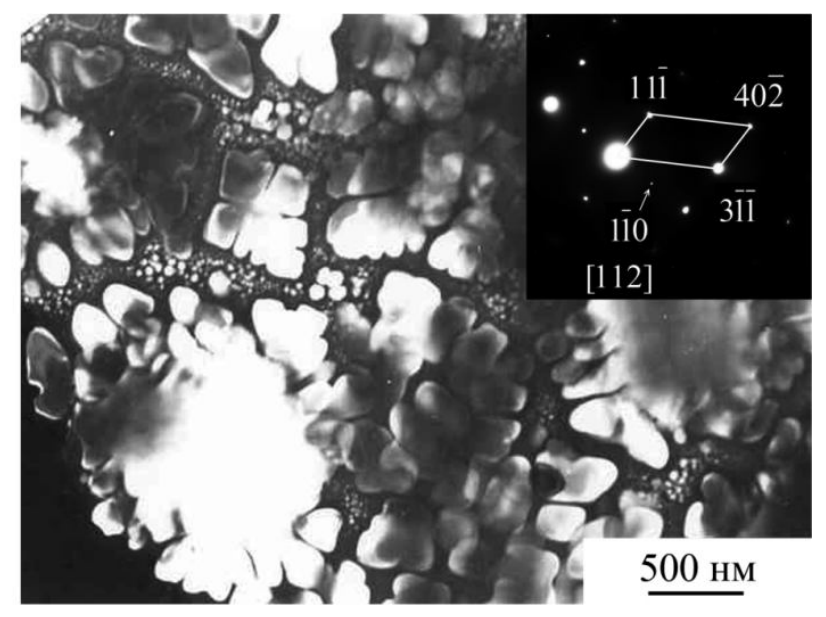

6

Рис. 1. Структура лопатки из сплава ЧС-70 после деформации: $a$ - структура пера лопатки, светлопольное изображение; $\sigma$ - замковая часть, темнопольное изображение в рефлексе $\gamma^{\prime}$-фазы (указан стрелкой)

В процессе эксплуатации замок подвергается в основном термическому воздействию, тогда как перо оказывается одновременно под действием растягивающих напряжений из-за центробежной силы и в условиях малоцикловой усталости из-за вибрации. Напряжения мак- 
симальны в спинке пера (участок выпуклой части пера с максимальной кривизной) и в центре вогнутой части пера вдоль оси растяжения.

В замковой части лопатки практически нет напряжений. Там происходит вызванная термическим воздействием коагуляция упрочняющей фазы, в то время как в пере лопатки повышена плотность дислокаций, сосредоточенных в основном в участках твердого раствора. После эксплуатации по стандартному режиму дефектов внутри частиц упрочняющей фазы не наблюдается.

После эксплуатации по форсированному режиму в спинке пера (выпуклая часть) и вогнутой части пера в области максимальных напряжений кроме высокой плотности дислокаций наблюдается большое количество дефектов упаковки внутри частиц $\gamma^{\prime}$-фазы. Тот факт, что дифракционный контраст от дефектов упаковки присутствует на темнопольных изображениях, полученных в рефлексе интерметаллидной фазы, показывает, что дефекты упаковки принадлежат интерметаллидной фазе (являются сверхструктурными) (рис. 2 б).

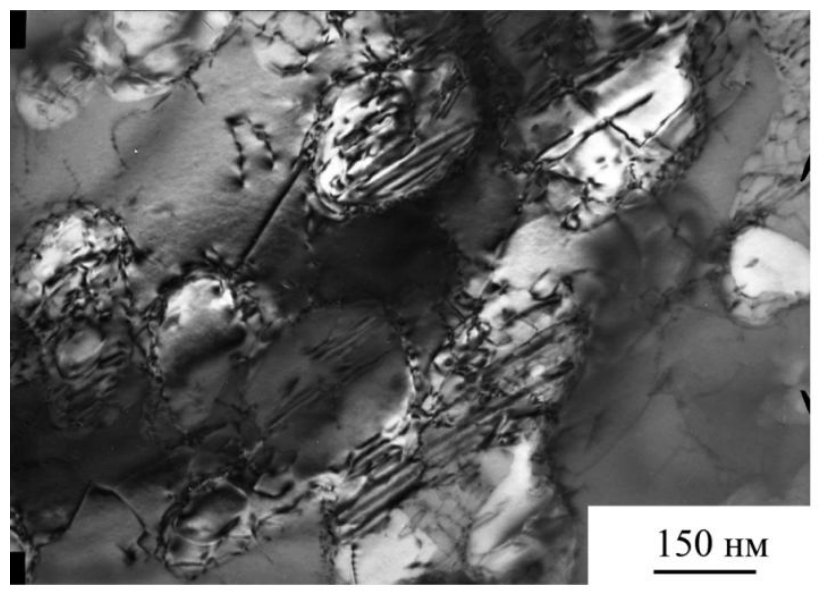

$a$

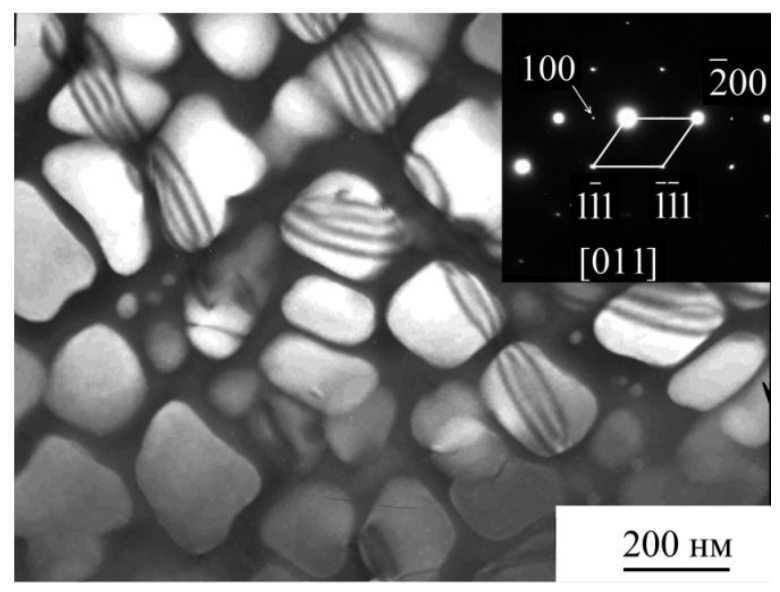

б

Рис. 2. Дефекты упаковки в частицах упрочняющей интерметаллидной фазы, спинка пера лопатки после деформации: $a$ - светлопольное изображение; $\sigma$ - темнопольное изображение в рефлексе $\gamma^{\prime}$-фазы

Результаты измерений полевой зависимости намагниченности $M(H)$, проведенных на тех же образцах, что были использованы для электронной микроскопии, приведены на рис. 3. Они коррелируют как с уровнем напряжений, так и с количеством дефектов кристаллической структуры в различных частях детали.

У образца, вырезанного из замка лопатки, зависимость $\mathrm{M}(\mathrm{H})$ линейна и практически совпадает с $\mathrm{M}(\mathrm{H})$ для исходного (без деформации) образца сплава ЧС-70. Эти образцы находятся в парамагнитном состоянии. Для образцов, вырезанных из лопатки в области спинки пера, зависимость $\mathrm{M}(\mathrm{H})$ имеет характер кривой с насыщением, значения намагниченности существенно выше, чем в исходном состоянии. Имеет место узкий гистерезис, площадь которого увеличивается одновременно с ростом намагниченности насыщения (рис. 3 б). Температурная зависимость магнитной восприимчивости в переменном магнитном поле приведена на рис 4. Можно видеть, что после эксплуатации по экспериментальному режиму на кривой кроме основного пика, связанного с температурой Кюри основного сплава, появляется второй пик вблизи $280 \mathrm{~K}$. 


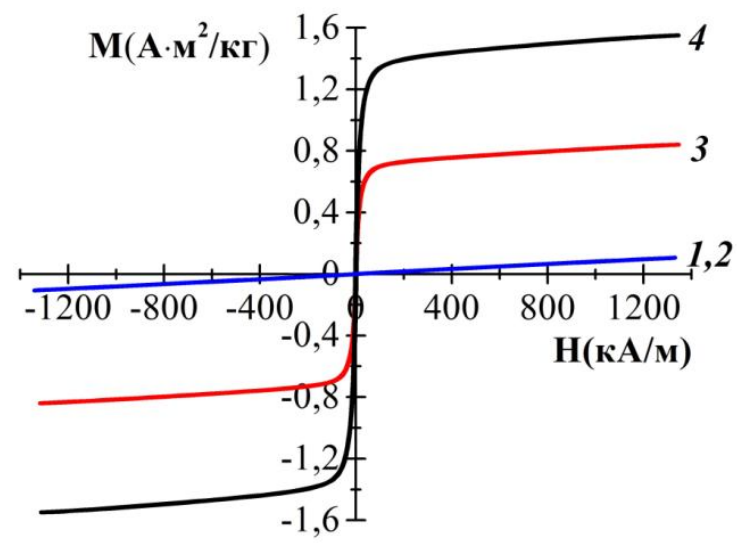

$a$

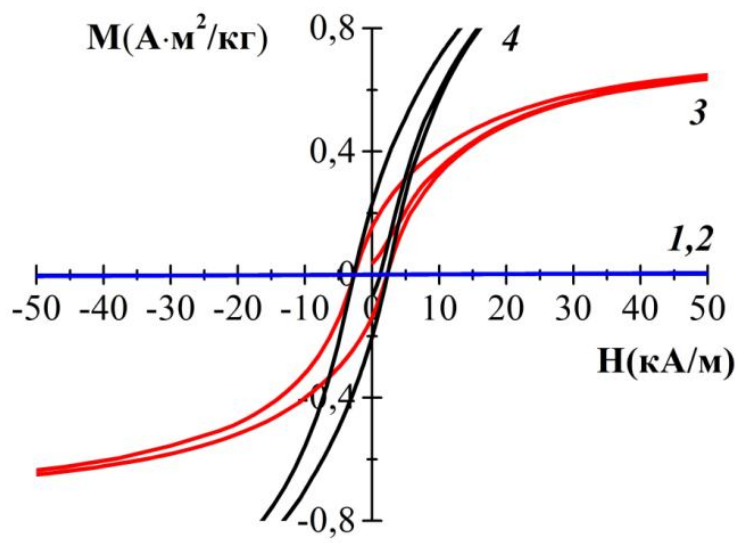

$\sigma$

Рис. 3. Магнитные свойства образцов сплава ЧС-70, вырезанных из различных частей турбинной лопатки: $a$ - полевая зависимость намагниченности $\mathrm{M}(\mathrm{H})$; $\sigma$ - центральная часть петли гистерезиса.

1 - исходный; 2 - замковая часть; 3 - вогнутая часть пера; 4 - спинка пера

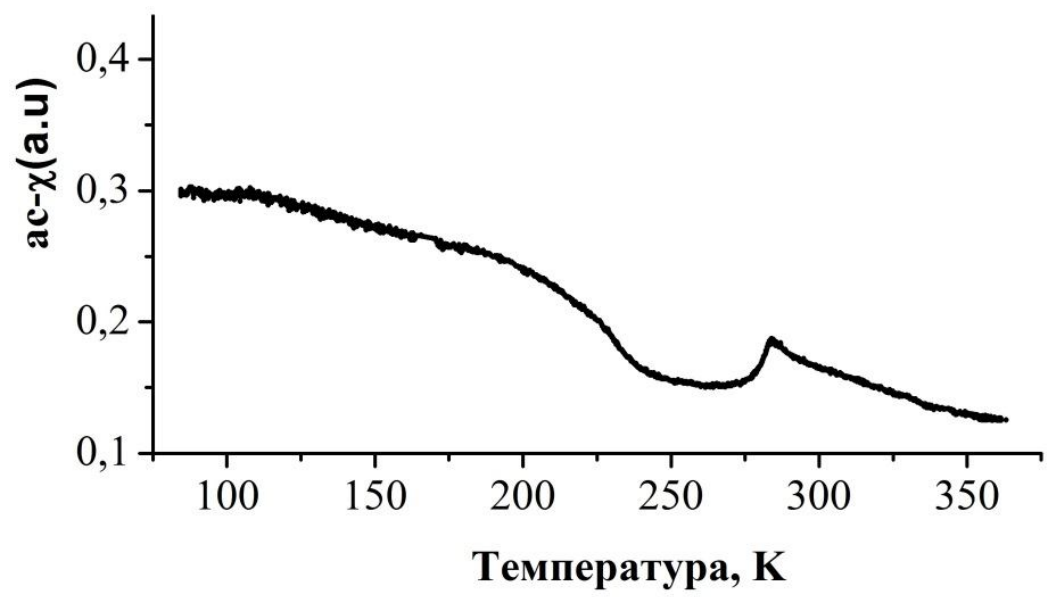

Рис. 4. Зависимость магнитной восприимчивости в переменном магнитном поле от температуры для образца, вырезанного из спинки пера турбинной лопатки

Материал турбинных лопаток после эксплуатации по стандартному режиму $\left(800{ }^{\circ} \mathrm{C}\right)$ в пределах гарантийного срока по техническим условиям (ТУ) остается в парамагнитном состоянии. При стандартном режиме, сколько бы дефектов не возникало внутри областей твердого раствора, дефекты внутри частиц $\gamma^{\prime}$-фазы отсутствуют в течение всего периода эксплуатации. Время эксплуатации по ТУ специально было выбрано так, чтобы избежать возникновения устойчивых комплексов дефектов внутри интерметаллида, поскольку их появление свидетельствует об его разупрочнении и является главным фактором деградации структуры сплава. Условием длительной высокотемпературной эксплуатации изделий из жаропрочного сплава также является его стабильность по отношению к фазовым превращениям. При этом у сплава не наблюдается появления со временем каких-либо ферромагнитных свойств.

После эксплуатации по форсированному режиму ферромагнитные свойства проявляются локально - в области максимальных напряжений вдоль оси пера лопатки, и их проявление связано, по-видимому, с появлением внутри частиц интерметаллидной фазы определенного типа дефектов, которыми могут быть взаимодействующие между собой дефекты упаковки. 


\section{1 Структура сплава ЧС-70 после высокотемпературных испытаний на растяжение}

Напряженное состояние, возникающее в турбинной лопатке в процессе эксплуатации, близко к одноосному растяжению. Поэтому были проведены испытания с активным нагружением цилиндрических образцов жаропрочного никелевого сплава ЧС-70 в области температур 880-920 ${ }^{\circ} \mathrm{C}$ со скоростью растяжения $8 \cdot 10^{-7} \mathrm{M} / \mathrm{c}(0,05 \mathrm{Mм} / \mathrm{Mин})$. На рис. 5 приведены кривые растяжения исследованных сплавов. В работе [9] были получены и подробно проанализированы такого типа кривые при высокотемпературной деформации никелевых жаропрочных сплавов. Низкая скорость растяжения создаёт условия для релаксации напряжений в ходе испытаний, т. е. приводит к резкому увеличению пластичности сплава и уменьшению прочностных свойств (табл. 2).

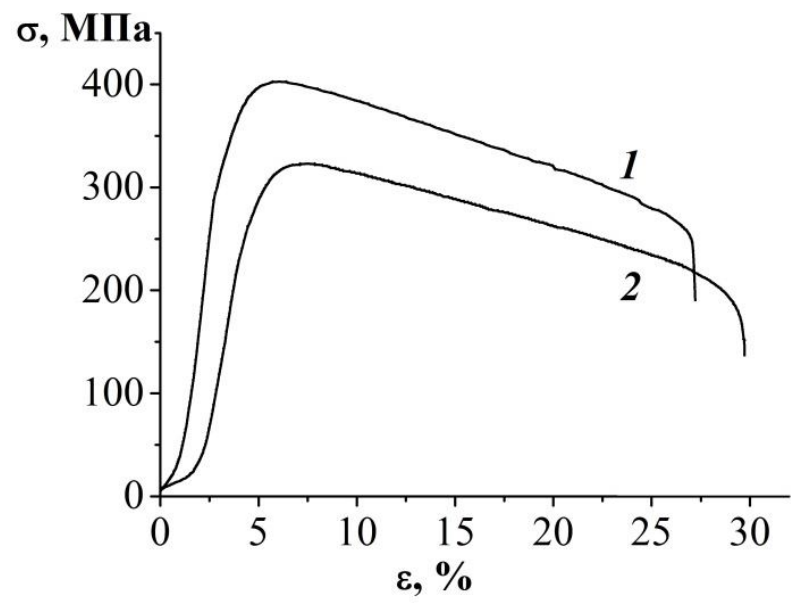

Рис. 5. Кривые растяжения образцов сплава ЧС-70:1 - при $880{ }^{\circ} \mathrm{C} ; 2$ - при $920{ }^{\circ} \mathrm{C}$

Структурное состояние образцов сплава ЧС-70, испытанного на растяжение при температурах $880{ }^{\circ} \mathrm{C}$ и $920{ }^{\circ} \mathrm{C}$, оказывается близким. В этих образцах наблюдалась структура динамического возврата, когда участки с высокой плотностью дислокаций перемежаются с участками субструктуры с малоугловыми границами.

Таблица 2 - Механические свойства сплава ЧС-70

\begin{tabular}{|c|c|c|c|c|c|}
\hline \multirow{2}{*}{$\mathrm{T}_{\text {исп }}{ }^{\circ} \mathrm{C}$} & \multicolumn{3}{|c|}{ Данные [10] } & \multicolumn{2}{c|}{$\begin{array}{c}\text { Данные настоящей } \\
\text { работы }\end{array}$} \\
\cline { 2 - 6 } & 20 & 850 & 900 & 880 & 920 \\
\hline$\sigma_{\mathrm{B}}, \mathrm{MПа}$ & 900 & 800 & 600 & 400 & 320 \\
\hline$\sigma_{0,2}, \mathrm{MПа}$ & 750 & 680 & 520 & 315 & 250 \\
\hline$\delta, \%$ & 3 & 6 & 2 & 26 & 29 \\
\hline
\end{tabular}

На рис. 6 приведено электронно-микроскопическое изображение структуры образцов после испытаний на растяжение. Можно видеть формирование блочной субструктуры, образованной малоугловыми границами с разворотом соседних фрагментов на 2-3․ 


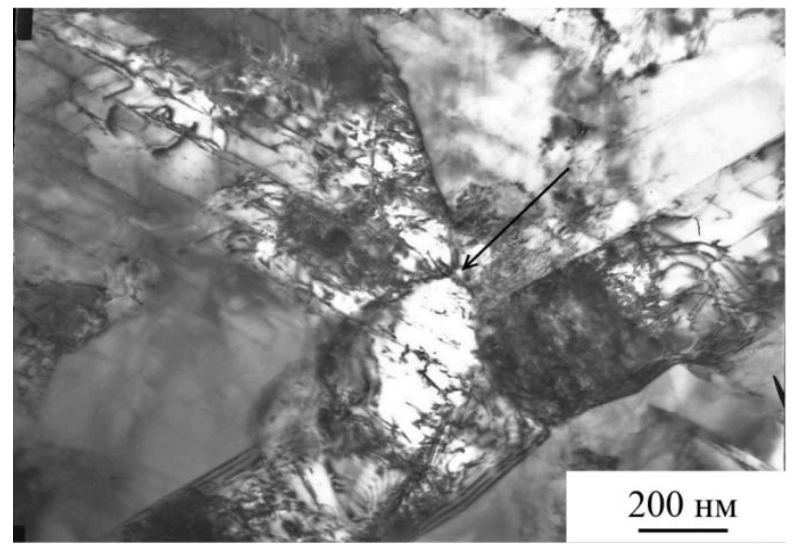

$a$

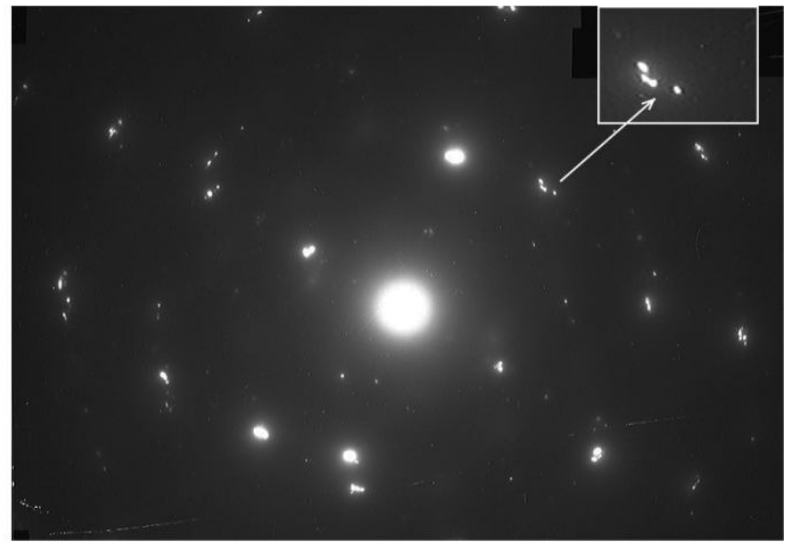

6

Рис. 6. Структура сплава после испытаний при $920{ }^{\circ} \mathrm{C}$ : $a$ - светлопольное изображение; $\sigma$ - дифракционная картина, снятая с тройного стыка зерен

Отражения, удаленные от центрального пятна, фрагментируются, поскольку образованы областями кристалла с малым разворотом друг относительно друга. Такая структура еще раз подтверждает, что процесс возврата динамический и проходит в ходе испытаний.

Релаксация напряжений при деформации в условиях динамического возврата сопровождается непрерывным рождением и аннигиляцией дислокаций, формированием и распадом субграниц. В структуре сплава ЧС-70 после высокотемпературного растяжения наблюдается повышенная плотность дислокаций, сосредоточенных, в основном, в областях твёрдого раствора. С ростом плотности дислокаций начинается фрагментация крупных частиц $\gamma^{\prime}-$ фазы, и частица оказывается разделена малоугловыми границами. На темнопольных изображениях появляется характерный контраст, когда в одном и том же сверхструктурном рефлексе частица светится не как целое, а различные ее участки загораются последовательно при наклоне объекта в колонне микроскопа (рис. 7).

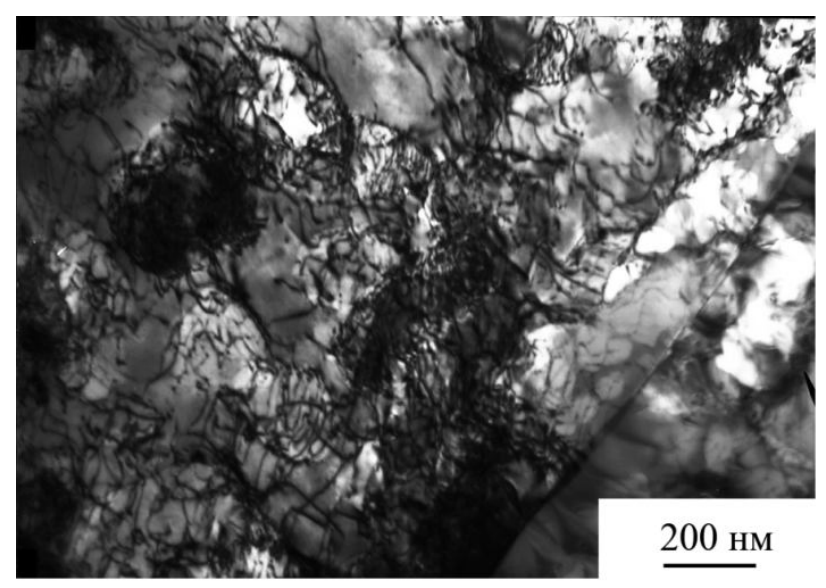

$a$

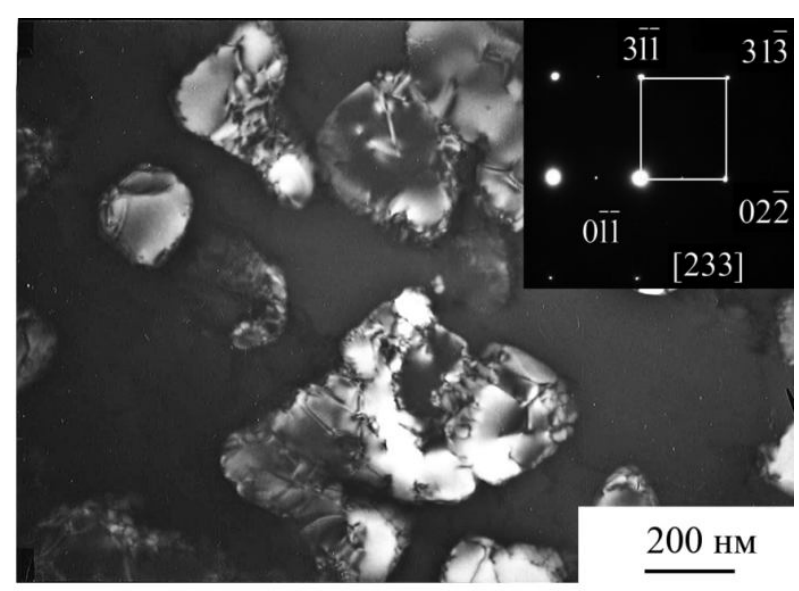

$\sigma$

Рис. 7. Структура сплава ЧС-70 после испытаний при $920{ }^{\circ} \mathrm{C}: a-$ дислокации внутри твердого раствора; $\sigma$ - фрагментация частиц $\gamma^{\prime}$-фазы, темнопольное изображение в рефлексе $\gamma^{\prime}$-фазы

Проведены измерения полевой зависимости намагниченности $M(H)$ для образцов после высокотемпературной деформации. При высокотемпературных испытаниях на растяжение с низкой скоростью нагружения изменения магнитных свойств не наблюдается, сплав остаётся в парамагнитном состоянии (рис. 8). 


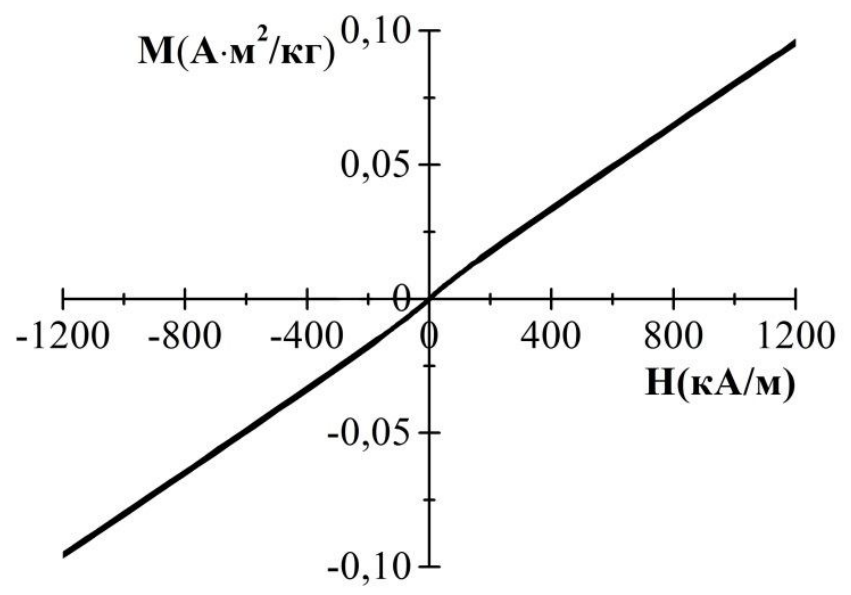

Рис. 8. Полевая зависимость намагниченности для образцов сплава ЧС-70 после высокотемпературных испытаний

В условиях динамического возврата не происходит формирования структуры, необходимой для появления ферромагнитных свойств, внутри частиц интерметаллида нет устойчивых комплексов дефектов, обладающих ферромагнитным порядком. Малоугловые границы такими дефектами не являются. При структурных исследованиях не выявлено образования сверхструктурных дефектов упаковки.

\section{2 Структура сплава ЧС-70 после деформации методом сдвига под давлением}

Формирование нанокристаллического состояния в образцах двойного интерметаллида $\mathrm{Ni}_{3} \mathrm{Al}$ после деформации кручением под давлением подробно исследовано [11, 12]. При малых углах поворота в наковальнях Бриджмена образуются фрагменты размером около 100 нм, разделенные малоугловыми границами. С увеличением степени деформации происходит локальное разупорядочение, образуется большое количество двойников деформации, увеличивается плотность дислокаций. Дальнейшее увеличение степени деформации приводит к полному разупорядочению интерметаллида. Частичное восстановление дальнего порядка начинается при нагреве до $350{ }^{\circ} \mathrm{C}$.

Большая пластическая деформация сплава ЧС-70 приводит к образованию субмикрокристаллической структуры, средний размер микрокристаллитов после деформации на 10 оборотов составлял 20 нм. Деформация методом сдвига под давлением приводит к образованию многочисленных дефектов внутри областей твердого раствора и в частицах интерметаллида, формированию большого числа границ и, соответственно, приграничных искаженных объемов (рис. 9). Все это сопровождается значительным падением степени дальнего порядка в интерметаллидной фазе сплава ЧС-70. Увеличение количества дефектов внутри твердого раствора приводит к увеличению микротвердости с 3 ГПа в исходном состоянии до 6,2 ГПа при 10 оборотах.

Подробный анализ образцов сплава ЧС-70 не выявил существенных изменений их магнитных свойств после деформации. Сплав остаётся в парамагнитном состоянии после деформации. Полученные результаты согласуются с работой [13], в которой установлено, что полное разупорядочение интерметаллида $\mathrm{Ni}_{3} \mathrm{Al}$ приводит к уменьшению магнитной восприимчивости в парамагнитном состоянии. 


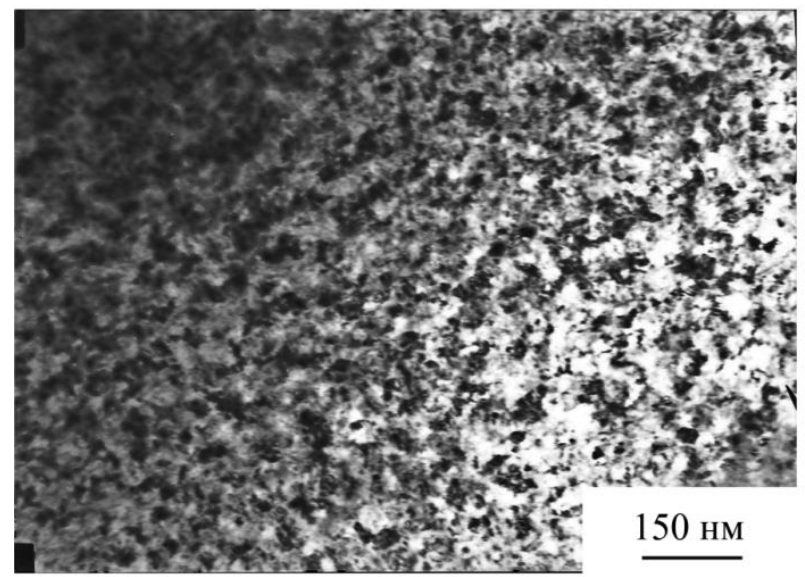

$a$

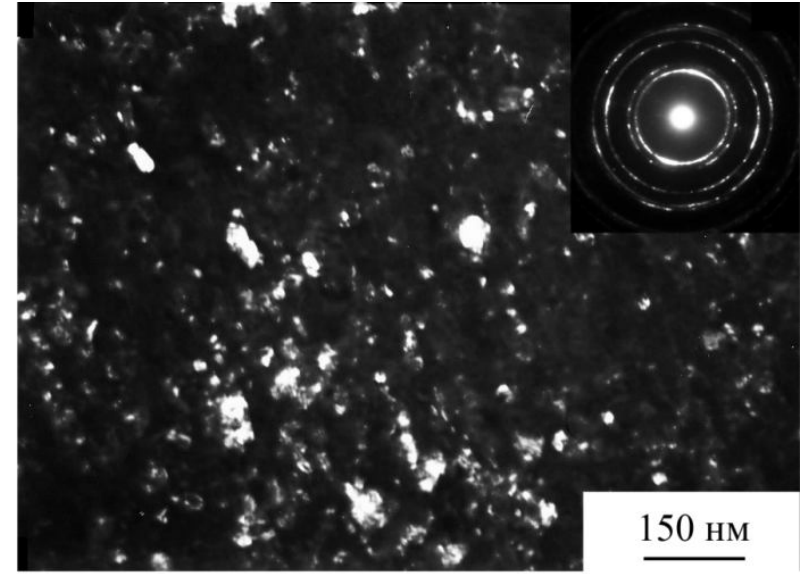

$\sigma$

Рис. 9. Микроструктура сплава после деформации 10 оборотов:

$a$ - светлопольное изображение; $\sigma$ - темнопольное изображение в матричном рефлексе

В качестве ферромагнитного кластера, по-видимому, выступает устойчивый комплекс дефектов внутри частицы интерметаллида, которая в целом сохраняет высокую степень дальнего порядка так, как это было при высокотемпературной деформации турбинной лопатки. Полное разупорядочение интерметаллида не меняет магнитное состояние. В результате деформации методом сдвига под давлением происходит снижение степени дальнего порядка в упрочняющей интерметаллидной фазе. После деформации сдвигом под высоким давлением на 10 оборотов происходит практически полное разупорядочение: на рентгеновской дифракции не регистрируются сверхструктурные пики (рис. 10), на электронограммах отсутствуют сверхструктурные отражения.

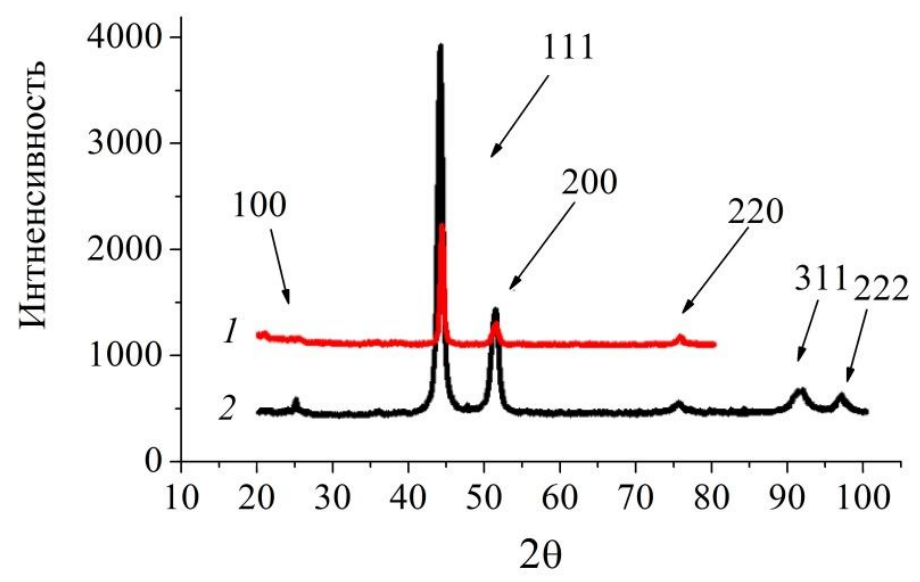

Рис. 10. Дифрактограммы образца сплава ЧС-70 после деформации методом сдвига под давлением (1) и в исходном состоянии (2)

\section{4. Заключение}

Длительная эксплуатация турбинной лопатки из сплава ЧС-70 по экспериментальному режиму с повышенным уровнем рабочих температур привела к появлению ферромагнитных свойств у исходно парамагнитного материала в участке детали с максимально высокими значениями рабочей температуры и напряжений. Повышение намагниченности насыщения происходит только в тех частях лопатки, где наблюдается большое количество дефектов упаковки внутри частиц интерметаллидной фазы $\left(\mathrm{Ni}_{3} \mathrm{Al}\right)$, например в спинке пера лопатки. В замко- 
вой части, где в процессе эксплуатации дефектов в $\mathrm{Ni}_{3} \mathrm{Al}$ не возникло, изменений магнитных свойств нет, сплав сохраняет парамагнитное состояние.

Высокотемпературные испытания на растяжение с низкой скоростью деформации сопровождаются динамическим возвратом. В условиях релаксации напряжений дефекты упаковки внутри интерметаллидных частиц не образуются. Основным элементом структуры являются малоугловые границы, появление которых не приводит к изменению магнитных свойств.

Деформация методом сдвига под давлением сопровождается значительным падением степени дальнего порядка в интерметаллидной фазе сплава ЧС-70, в результате образец остаётся парамагнитным.

Таким образом, различный характер изменения магнитных свойств определяется различием структуры, сформированной в результате деформации.

При сравнении дефектного состояния и магнитных свойств образцов, полученных при деформации по различным схемам, можно утверждать, что изменение магнитных свойств никелевых жаропрочных сплавов при высокотемпературной деформации действительно связано с интерметаллидной фазой.

\section{Благодарность}

Работа выполнена в рамках государственного задания ФАНО России (тема «Кристалл», № 01201463333) при частичной поддержке РФФИ (проект № 14-03-31499).

\section{Литература}

1. Stoloff N. S. Physical and mechanical metallurgy of $\mathrm{Ni}_{3} \mathrm{Al}$ and its alloys // International Materials Reviews. - 1989. - Vol. 34, iss. 4. - P. 153-184.

2. Formation of nanosized ferromagnetic phases during plastic deformation and subsequent annealing of stable austenitic steels / A. I. Deryagin, V. A. Zavalishin, V. V. Saragadze, B. M. Efros // Russian Journal of Nondestructive Testing. - 2007. - Vol. 43, iss. 7. - P. 427-435. DOI: $10.1134 /$ S1061830907070029.

3. Rhee Joo Yull, Kudryavtsev Y. V., Lee Y. P. Optical, magneto-optical, and magnetic properties of stoichiometric and off-stoichiometric $\gamma^{\prime}$-phase $\mathrm{Ni}_{3} \mathrm{Al}$ alloys // Physical Review B. - 2003. Vol. 68, iss. 4. - P. 045104-1-045104-8. - DOI: 10.1103/PhysRevB.68.045104.

4. Magnetic effects of structural disorder in the itinerant ferromagnet $\mathrm{Ni}_{3} \mathrm{Al}$ studied by magnetic and neutron methods on stoichiometric and off-stoichiometric samples / B. Idzikowski, Y. V. Kudryavtsev, Y. H. Hyun, Y. P. Lee, J. Klenke // Journal of Alloys and Compounds. - 2006. - Vol. 423, iss. 1-2. - P. 267-273. - DOI: 10.1016/j.jallcom.2006.01.088.

5. Zeng Q., Baker I. The effect of local versus bulk disorder on the magnetic behavior of stoichiometric $\mathrm{Ni}_{3} \mathrm{Al} / /$ Intermetallics. - 2007. - Vol. 15, iss. 3. - P. 419-427. DOI: 10.1016/j.intermet.2006.08.010.

6. Umakoshi Y., Hiroyuki Y., Toshifumi Y. Quantitative analysis of $\gamma$ (gamma) precipitate in cyclically deformed $\mathrm{Ni}_{3}(\mathrm{Al}, \mathrm{Ti})$ single crystals using magnetic technique // Proc. MRS Fall Meeting, Boston, USA, November 28 - December 3, 2004. - 2004. - Vol. 842. - P. 2.3.1 - 2.3.6.

7. The structure and magnetic properties of a heat-resistant nickel-base alloy after a hightemperature deformation / N. N. Stepanova, D. I. Davydov, A. P. Nichipuruk, M. B. Rigmant, N. V. Kazantseva, N. I. Vinogradova, A. N. Pirogov, E. P. Romanov // The Physics of Metals and Metallography. - 2011. - Vol. 112, iss. 3. - P. 309-317. - DOI: 10.1134/S0031918X11030288.

8. Structure property correlation study of a service exposed first stage turbine blade in a power plant / A. K. Ray, S. R. Singh, J. Swaminathan, P. K. Roy, Y. N. Tiwari, S. C. Bose, R. N. Ghosh // Materials Science and Engineering: A. - 2006. - Vol. 419, iss. 1-2. P. 225-232. - DOI: 10.1016/j.msea.2005.12.030. 
9. Левит В. И., Смирнов М. А. Высокотемпературная термомеханическая обработка аустенитных сталей и сплавов. - Челябинск : ЧГТУ, 1995. - 276 с.

10. Скуднов В. А., Тарасенко Ю. П., Бердник О. Б. Выбор оптимальной рабочей температуры никелевых сплавов ЧС70-ВИ и ЧС88У-ВИ с позиции синергетики // Технология металлов. -2008 . - № 12. - С. 16-20.

11. The mechanism of nanocrystalline structure formation in $\mathrm{Ni}_{3} \mathrm{Al}$ during severe plastic deformation / A. V. Korznikov, G. Tram, O. Dimitrov, G. F. Korznikova, S. R. Idrisova, Z. Pakiela // Acta Materialia. - 2001. - Vol. 49, iss. 4. - P. 663-671. - DOI: 10.1016/S1359-6454(00)00345-1.

12. Evolution of defect substructure in the $\mathrm{Ni}_{3} \mathrm{Al}$ alloy in the course of severe plastic deformation by torsion under pressure / A. N. Tyumentsev, M. V. Tretyak, Yu. P. Pinzhin, A. D. Korotaev, R. Z. Valiev // Fizika Metallov i Metallovedenie. - 2000. - Vol. 90, iss. 5. - P. 44-54.

13. Effect of a nanosized state on the magnetic properties of $\mathrm{Ni}_{3}(\mathrm{Al}, \mathrm{Fe})$ and $\mathrm{Ni}_{3}(\mathrm{Al}, \mathrm{Co}) /$ N. V. Kazantseva, V. P. Pilyugin, V. A. Zavalishin, N. N. Stepanova // The Physics of Metals and Metallography. - 2014. - Vol. 115, iss. 3. - P. 243-247. - DOI: 10.1134/S0031918X14030053. 